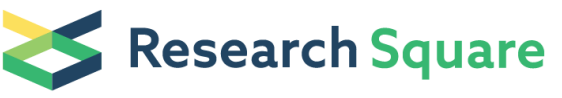 \\ Preprints are preliminary reports that have not undergone peer review. \\ They should not be considered conclusive, used to inform clinical practice, \\ or referenced by the media as validated information.
}

\section{A Practical Triage and Risk Scoring for Prediction of Early Mortality in Polytrauma Patients: GAS-TRS}

Tian Xie

Huazhong University of Science and Technology Union Shenzhen Hospital

\section{Shikai Wang}

Huazhong University of Science and Technology Union Shenzhen Hospital

\section{Nan Du}

Huazhong University of Science and Technology Union Shenzhen Hospital

\section{Qunxing Huang}

Huazhong University of Science and Technology Union Shenzhen Hospital

Jianguo Wu

Huazhong University of Science and Technology Union Shenzhen Hospital

Xiaoqiao Mo

Shanghai Jiao Tong University School of Medicine

\section{Xiangda Zhang}

Monash University SPHPM: Monash University School of Public Health and Preventive Medicine

Jiayuan Tu

Nanjing University

Xinsheng Cheng ( $\nabla$ shenzhenhos_21@sina.com)

Huazhong University of Science and Technology Union Shenzhen Hospital

\section{Research Article}

Keywords: Polytrauma, Mortality risk, Nomogram model, Artificial neural network, Predictive scoring system

Posted Date: December 6th, 2021

DOI: https://doi.org/10.21203/rs.3.rs-1086645/v1

License: @ (i) This work is licensed under a Creative Commons Attribution 4.0 International License. Read Full License 


\section{Abstract}

Background Accurate evaluation of mortality risk in polytrauma patients is crucial for guiding the precision treatment strategy. There are few scales designed to provide an early assessment of mortality risk. However, the complexity of available scoring systems limits their application in pre-hospital care. Here, we established a GAS-TRS system to estimate the risk of early death for individual polytrauma patients and assess the early mortality risk in the individual patient.

Methods We performed a secondary analysis from public Database. RCS and Multivariate Logistic regression analyses were used to screen potential prognostic factors for nomogram model. The VIF method examined multicollinearity, and VIF $\geq 5$ suggested multicollinearity in this model. CMA was used to characterize the causality relationship in nomogram model. A four-layer back-propagation artificial neural network (BP-ANN) model was built by neuralnet package on $\mathrm{R}$ software. AUC of ROC analysis or F1 score were used to analyze the quality of predictive performance of GAS-TRS system. DCA and precision-recall curves were used to make up for the limitations of ROC curves.

Results A total of 2406 patients were included in this analysis. Logistic regression analysis predicted four independent risk factors for nomogram model, including age (OR=1.03, 95\%Cl:1.02 1.03), GCS (OR=0.83, 95\%Cl:0.79 0.86), BE $(\mathrm{OR}=0.95,95 \% \mathrm{Cl}: 0.91 \sim 0.99)$ and serum lactic acid $(\mathrm{OR}=1.30,95 \% \mathrm{Cl}: 1.20 \sim 1.41)$ with an AUC of 0.88. Causal mediation analysis performed the mediation effect that lactate, age and BE accounted for $1.7 \%, 0.7 \%$ and $3.0 \%$ indirect effect.The calibration curve showed model has good highly consistent with actual condition after bootstrapping. DCA showed the net benefit probability was between $2 \%$ and $85 \%$ and could bring more benefits for predicting early mortality. Then the input neurons were selected step by step in BP-ANN model. An optimal BP-ANN with an AUC of 0.91and AUPRC of 0.79 was established.

Conclusion We established a GAS-TRS predictive system which includes a quick prognostic nomogram model and a precise BP-ANN model to evaluate early mortality within 72 hours for polytrauma patients. This scoring system might be practical and more efficient in identifying high-risk polytrauma patients. Moreover, this system may also guide triaging and precise initial individual treatment strategy for pre-hospital medical personnel.

\section{Background}

Trauma injury remains a significant burden to global healthcare system, especially in low- and middle-income countries $^{[1]}$. Noticeably, during $2000-2010$, trauma-related mortality increased by $22.8 \%$ in the US ${ }^{[2]}$ and by $24 \%$ worldwide $(1990-2010)^{[3]}$. Unintentional injury and violence-associated trauma contributed to approximately 4,000,000 people deaths worldwide, and leaving tens of millions of people permanently disabled each year ${ }^{[1]}$. While many of these deaths occurred at the scene of the trauma, around $44 \%$ of patients died after admission to hospital ${ }^{[4]}$.

Early evaluation of the pathophysiological status of traumatic patients is pivotally important for intensive care management and surgical decision-making ${ }^{[5]}$. Triage system may provide a scale for identifing patients at high risk and offers early alerts for initiation of appropriate strategies for preventable trauma-associated death ${ }^{[6]}$. However, the most frequently used trauma scores categorized as anatomical or physiological are either too rough for the prediction of early death or too complicated and time-consuming for early usage in trauma patients. Existing prognostic models (biomarkers or scoring systems) mainly focus on general hospital mortality or four weeks in-hospital death ${ }^{[1,7]}$. In practice, data from a Level 1 trauma center revealed that $26 \%$ of all in-hospital deaths (including dead on arrival, DOAs) occurred within one hour of hospital arrival, $59 \%$ within 24 hours and $78 \%$ by day three while $98 \%$ occurred within 30 days of injury ${ }^{[8]}$. Thus, more accurate prognostic models for $72 \mathrm{~h}$ death are in need for pre-hospital and in-hospital trauma management. 
Initial serum lactate and lactate clearance, serum procalcitonin (PCT) and scoring tools are capable of early prediction in critically injured patients ${ }^{[9-12]}$. Yet, mortality in patients with the same single predictive parameter may range due to patients' physical quality or status. Moreover, prognostic models in hand for predicting early mortality in polytrauma patients are limited and non-specific, and there are few extensive samples studies available now. In this study, we aimed to develop a simple prognostic Nomogram model that could be used at the point of care to estimate the risk of early death for individual polytrauma patient in pre-hospital care. Thus, we promote an effective triage strategy and initiated active trauma management according to Precision Medicine. Moreover, after triage to proper care management and complete evaluation of polytrauma patients, a more accurate predicting model was established to further assess the early mortality risk in the individual patient.

\section{Material And Methods}

\section{Study design and data source}

This retrospective population study was conducted using unidentified data from the DRYAD database (https://datadryad.org), an open-access public database, obtained from Dr. Halvachizadeh, University Hospital Zurich, Zurich, Switzerland, with formal authorization ${ }^{[13]}$. Original studies were approved by regional institutional review board, and their public data included the anonymized patients which did not require further patient consent ${ }^{[14]}$. The aims of those studies focused on the assessment of Berlin Definition and how to detect a polytrauma patient at risk of complications. In brief, Dr. Halvachizadeh enrolled 3668 adult polytrauma patients treated between 1999 and 2006 in University Hospital Zurich. Polytrauma patients in their studies were defined as having an ISS greater than or equal to 16,

along with the criteria of the Berlin definition ${ }^{[14]}$. Patients were randomly split into a training cohort and another separate validation set at a ratio of 7:3. Cohorts were divided into dead and alive groups according to the status at 72 hours after admission. The flowchart of enrolled patients is shown in Figure1.

\section{Clinical data}

The following basic patient data were extracted from datasets above: age, sex, length of stay in hospital, length of stay in intensive care unit, body mass index (BMI), injury severity score (ISS), Glasgow coma scale (GCS), arterial blood gas analysis ( $\mathrm{pH}$, base excess and lactic acid level) and 72-hour mortality. Samples missed any one of the parameters above would be removed. All parameters were recorded at admission and ISS and GCS were presented as total scores without details.

\section{Restricted cubic splines}

Restricted cubic splines (RCS) had been widely described as a valid strategy to analyze the relationship between independent variables and mortality. RCS is a smoothly joined sum of polynomial functions that provide greater flexibility for fitting and modeling, especially complex relationships. Additionally, RCS can identify the risk function inflection point in polynomial functions. In this study, RCS was used to explore the trend between independent variables and mortality. The spline was defined using three knots(the 5th, 50th, and 95th percentiles), and threshold was determined as the point in time with the smallest hazard ratio (HR). The $95 \% \mathrm{Cl}$ was derived by bootstrap resampling.

\section{Nomogram Model Building}

After RCS analysis the relationship between independent variables and mortality, multi-variate Logistic regression analyses were used to screen potential prognostic factors. Factors with p-values, which were calculated based on univariate Logistic regression models, over 0.1 were removed from the associated models. The variance inflation factor (VIF) method examined multicollinearity, and VIF $\geq 5$ suggested multicollinearity in our model. The predictive 
performance of nomogram and other models to predict the survival were quantified using the area under the curve (AUC) of the receiver operating characteristic (ROC) analysis or the consistency index (C-index) and comprehensively evaluated based on the training set and validation set, respectively. The performance of the nomogram was also performed with calibration. Moreover, the clinical utilities of the nomogram were also carefully investigated using decision curve analysis (DCA) to make up for the limitations of ROC curves.

\section{Causal mediation analysis}

Causal mediation analysis(CMA) is a method to differentiate the total effect of treatment into direct and indirect effects. The indirect effect on the outcome is mediated via a mediator ${ }^{[15]}$. The analysis produces an average causal mediation effect (ACME), average direct effect (ADE) and total effect. To explore whether the effect of variables in our nomogram model was proportionally mediated by each other, we used CMA to characterize the causality relationship in our retrospective study.

\section{BP-ANN Model Building}

A four-layer back-propagation artificial neural network (BP-ANN) model was built by neuralnet package on R software (version 4.0.0). In brief, an initial essential three-layer BP-ANN model input neurons were selected based on nomogram model. More input neurons were added according to independent risk factors or clinical decision-making strategies to achieve better performance. After input neurons were confirmed, hide layers and activation functions were optimized (Figure 1). Receiver operator curves (ROCs), precision, F1 Score and the area under the precision-recall curve (AUPRC)were generated for the validation datasets. Optimal BP-ANN model were established based on parameters above as the evaluation of model performance.

\section{Statistical analysis}

Continuous variables were evaluated for normal distribution using kurtosis and skewness. Normally distributed continuous variables were presented as mean \pm standard deviation and compared using Student t-tests. Skewed data were expressed as median and interquartile range (IQR) compared with Wilcoxon test. Categorical variables were presented as numbers (percentage) and compared using the chi-squared test. Univariate and multivariate logistic regression analyses were performed to identify the potential covariates associated with 72-hour mortality. All analyses were performed on R software (version 4.0.0) and a p-value smaller than 0.05 was considered statistically significant.

\section{Results}

\section{Characteristics and risk factors in polytrauma patients}

A total of 2406 patients who suffered polytrauma were included for analysis after excluding those with missing values. Of whom 1800 (74.8\%) were males, and the mean age of polytraumatic patients was $44.4 \pm 19.9$ years old. 491 individuals were dead within 72 hours after admission. The median total length of stay was 13 [4.00;24.8] days, while median length of stay in ICU was $5[2.00 ; 12.0]$ days. Table 1 showed demographic and clinical characteristics of the enrolled polytrauma patients. 
Table 1

Demographic and clinical characteristics of polytrauma patients according to their 72-hour survival status.

\begin{tabular}{|c|c|c|c|c|c|}
\hline & \multirow[t]{2}{*}{ All } & \multirow[t]{2}{*}{ Train set } & \multicolumn{2}{|l|}{ Train set } & \multirow[t]{2}{*}{$\mathrm{P}$ value (overall) } \\
\hline & & & Alive $\mathrm{N}=1336$ & Dead N=350 & \\
\hline $\mathrm{N}$ & 2406 & 1686 & 1336 & 350 & \\
\hline Age, years & $44.4 \pm 19.9$ & $44.4 \pm 20.1$ & $43.1 \pm 19.2$ & $49.6 \pm 22.6$ & $<0.001$ \\
\hline Gender & & & & & 0.540 \\
\hline $\begin{array}{l}\text { Female: } n \\
(\%)\end{array}$ & $606(25.2)$ & $429(25.4)$ & $335(25.1)$ & $94(26.9)$ & \\
\hline Male: n (\%) & $1800(74.8)$ & 1257 (74.6) & 1001 (74.9) & $256(73.1)$ & \\
\hline $\mathrm{BMI}, \mathrm{Kg} / \mathrm{m}^{2}$ & $25.1 \pm 4.40$ & $25.0 \pm 4.30$ & $25.3 \pm 4.21$ & $25.0 \pm 4.31$ & 0.481 \\
\hline ISS, points & $26.0[18.0 ; 36.0]$ & $26.0[18.0 ; 38.0]$ & $25.0[17.0 ; 34.0]$ & $34.0[25.0 ; 50.0]$ & $<0.001$ \\
\hline NISS, points & $34.0[27.0 ; 50.0]$ & $35.0[27.0 ; 50.0]$ & $34.0[25.0 ; 50.0]$ & $25.0[17.0 ; 34.0]$ & $<0.001$ \\
\hline GCS: points & $5.00[3.00 ; 15.0]$ & $4.00[3.00 ; 14.0]$ & $11.0[3.00 ; 15.0]$ & $3.00[3.00 ; 3.00]$ & $<0.001$ \\
\hline $\mathrm{pH}$ & $7.34[7.27 ; 7.39]$ & $7.34[7.27 ; 7.38]$ & $7.35[7.29 ; 7.39]$ & $7.29[7.18 ; 7.36]$ & $<0.001$ \\
\hline $\mathrm{BE}, \mathrm{mmol} / \mathrm{L}$ & $\begin{array}{l}-3.00[-5.90 \\
-0.80]\end{array}$ & $\begin{array}{l}-3.10[-6.00 \\
-0.90]\end{array}$ & $\begin{array}{l}-2.60[-5.10 \\
-0.50]\end{array}$ & $\begin{array}{l}-5.80[-10.28 \\
-2.30]\end{array}$ & $<0.001$ \\
\hline Lac, mmol/L & $2.30[1.40 ; 3.57]$ & $2.30[1.41 ; 3.60]$ & $2.10[1.30 ; 3.14]$ & $3.40[2.20 ; 6.18]$ & $<0.001$ \\
\hline Death & 491(20.4) & $350(20.8)$ & - & - & \\
\hline
\end{tabular}

Patients with early mortality within 72 hours presented with elder age, higher ISS, blood lactic acid level, lower GCS, pH and base excess level. Apart from gender, all the factors had statistically significant differences between groups $(P<0.001)$, which indicated that age, evaluation scales of trauma and arterial blood gases $(A B G s)$ test results could be potential risk factors for triaging high risk of mortality in polytraumatic patients (Table 1).

Next, RCS was performed to evaluate the relationship between independent variables and mortality rigorously. Figure 2 showed the trend of ORs for all-cause early mortality in polytrauma patients associated with the change of age, $\mathrm{pH}, \mathrm{base}$ excess (BE), GCS, and blood lactate acid, produced by the fully adjusted models incorporating 3-knot restricted cubic splines. The models produced no indication of significant nonlinear associations of mortality with age, $\mathrm{pH}, \mathrm{BE}, \mathrm{GCS}, \mathrm{or}$ blood lactate acid on admission.

The multivariate logistic regression analysis was performed to quantify the association between early death within 72 hours and the practical predictive factors. The independent risk factors were: age (OR=1.02, 95\% $\mathrm{Cl}: 1.02 \sim 1.03)$, GCS $(\mathrm{OR}=0.80,95 \% \mathrm{Cl}: 0.77 \sim 0.83), \mathrm{BE}(\mathrm{OR}=0.95,95 \% \mathrm{Cl}: 0.91 \sim 0.99)$ and serum lactic acid (OR=1.27, 95\%Cl:1.18 1.38) (Figure 3).

\section{ABGs-Age-GCS based Triage and Risk Scoring system (GAS-TRS) for quick triaging high risk in polytraumatic patients}

After selecting independent risk factors, we established a logistic regression model for the prediction of early mortality within 72 hours in polytraumatic patients. There was no evidence for collinearity after evaluating the VIF values, which were all smaller than 2 after categorized variables. Causal mediation analysis was performed to exclude the mediation effect. Lactate, age and BE accounted for only $1.7 \%, 0.7 \%$ and $3.0 \%$ indirect effect (Figure $4 A$, B and C). 
Then the included variables were applied to develop a predictive Nomogram for early mortality (Figure 5), which can be simply used for individualized prediction based on easily available indicators. The ROC analysis further demonstrated the predictive values in different models. And the AUCs of nomogram model, GCS scoring, ISS scoring and NISS scoring were $0.84,0.74,0.66$ and 0.76 , respectively, which indicated a better performance in prognostic prediction than commonly used tools, and the sensitivity and specificity of nomogram model were 0.84 and 0.84 , respectively (Figure 6A). Next, we performed a calibration test with a calibration plot, which showed good highly consistent with actual condition after bootstrapping for 500 times(Figure 6B). Then the DCA analysis was performed to access the optimal scoring systems in clinical use. DCA showed that the net benefit probability was between $2 \%$ and $85 \%$ and could bring more benefits for predicting the early mortality for polytraumatic patients, which was higher than GCS, ISS and NISS scoring (Figure 6C). The internal validation showed good performance with an AUC of 0.84 (Figure $6 \mathrm{~A}$ ). For better application of our nomogram model, a web version of our nomogram model tool was established at https://tientse523.shinyapps.io/Polytrauma/.

\section{GAS-TRS for identifying high risk in polytraumatic patients}

Although our nomogram GAS-TRS showed sound performance in predicting early mortality within 72 hours and was useful in quick triaging high risk polytraumatic patients, we further explored a more precise model for those polytraumatic patients when they were admitted to hospital. First, we performed logistic regression to identify independent risk factors, extending to complicated scoring including ISS and NISS scoring. Age (OR=1.03, 95\%Cl:1.02 1.03), GCS (OR=0.83, 95\% Cl:0.79 0.86), NISS scoring (OR=1.05, 95\%Cl:1.04 1.06) and serum lactic acid $(\mathrm{OR}=1.30,95 \% \mathrm{Cl}: 1.20 \sim 1.41)$ were identified as independent risk factors for prediction of early mortality in polytraumatic patients after admission(Figure 7). Next, we explored for optimal BP-ANN model based on independent risk factors step by step, and process of establishment of optimal BP-ANN model was shown in Figure 1 and Table 2. Eventually.

Table 2

Demographic and clinical characteristics of polytrauma patients according to their 72-hour survival status.

\begin{tabular}{|c|c|c|c|c|c|c|c|}
\hline $\begin{array}{l}\text { Variables in BP-ANN } \\
\text { Model }\end{array}$ & Cutoff & Sensitivity/Recall & Specificity & AUC & PPV/Precision & $\begin{array}{l}\text { F1 } \\
\text { Score }\end{array}$ & AUPRC \\
\hline Age+GCS+BE+Lact & 0.252 & 0.778 & 0.769 & 0.858 & 0.526 & 0.312 & 0.624 \\
\hline Age+GCS+BE+Lact +NISS & 0.248 & 0.809 & 0.780 & 0.882 & 0.517 & 0.622 & 0.678 \\
\hline Age+GCS+BE+Lact +ISS & 0.189 & 0.766 & 0.862 & 0.895 & 0.490 & 0.624 & 0.729 \\
\hline Age+GCS+BE+Lact $+p H$ & 0.182 & 0.686 & 0.886 & 0.873 & 0.425 & 0.573 & 0.678 \\
\hline Age+GCS+BE+Lact +Sex & 0.186 & 0.689 & 0.883 & 0.872 & 0.426 & 0.574 & 0.657 \\
\hline $\begin{array}{l}\text { Age+GCS+BE+Lact } \\
+ \text { ISS+Sex }\end{array}$ & 0.292 & 0.858 & 0.779 & 0.898 & 0.588 & 0.669 & 0.712 \\
\hline $\begin{array}{l}\text { Age+GCS+BE+Lact } \\
+\mathrm{ISS}+\mathrm{Sex}+\mathrm{pH}\end{array}$ & 0.215 & 0.808 & 0.831 & 0.904 & 0.529 & 0.647 & 0.727 \\
\hline $\begin{array}{l}\text { Logistics regression } \\
\text { model }(\mathrm{Age}+\mathrm{GCS}+\mathrm{BE}+\text { Lact } \\
+ \text { ISS+Sex+pH) }\end{array}$ & 0.201 & 0.769 & 0825 & 0.877 & - & - & - \\
\hline $\begin{array}{l}\text { Age+GCS+BE+Lact } \\
+ \text { +ISS+Sex+pH ( } 2 \text { hidden } \\
\text { layers) }\end{array}$ & 0.225 & 0843 & 0.840 & 0.913 & 0.583 & 0.687 & 0.788 \\
\hline Validation Set & 0.180 & 0.779 & 0.773 & 0.841 & 0.468 & 0.566 & 0.544 \\
\hline
\end{tabular}


7 BP-ANN models were established, and BP-ANN model with age, GCS, BE, Lactate, ISS, sex and pH was selected and compared with the same variables in logistics regression model, which showed better performance in BP-ANN than logistics regression model. Next, different hidden layers were explored to to optimize our BP-ANN model to majorize the best BP-ANN model. Finally, a BP-ANN model with 7 input neurons (age, GCS, be, lact, ISS, sex and pH) and 2 hidden layers with 6 and 3 neurons was established (Figure 8). The AUC of our optimal BP-ANN model was 0.91 with an F1 score of 0.69 (Table 2). Internal validation showed a better AUC of 0.84 with an F1 score of 0.57 . Precision-recall curves were also performed to assess the effectivity of BP-ANN model further and the AUPRCs were 0.79 in train set and 0.54 in validation set, respectively(Figure 10A and B).

\section{Discussion}

In this study, we applied nomogram methods to establish a quick and practical approach of prediction in early mortality for polytrauma patients, which was named as GAS-TRS system, and then improve the predictive performance of GASTRS with BP-artificial neural network (BP-ANN) algorithm. The nomogram model together with a web version (https://tientse523.shinyapps.io/Polytrauma/), had significantly better AUC than the classic ISS or NISS scoring. In addition, the nomogram model showed excellent calibration and in the decision curve analysis achieved a largest number of appropriate triages considering a trade-off with over-triages among all scorings, which means the greatest net benefit across the range of clinical thresholds. Further BP-ANN model with a complex scoring variable had significantly better sensitivity (recall) and PPV (precision) than the original GAS-TRS.

It is vital to early evaluate the mortality risk for polytrauma patients. In our study, polytrauma patients were defined as having an ISS greater than or equal to 16 , along with the criteria of the Berlin definition ${ }^{[16]}$. Although ISS or NISS scores are commonly used in polytrauma patients, there are anatomical tools with complicated calculations. Consist with previous studies, NISS score does overperform than ISS score ${ }^{[17-19]}$. However, ISS score is superior to NISS score in net benefit between the threshold probability of 0.5 to 0.7 with decision curve analysis in our study. That is to say, with the threshold probability between 0 and 0.5 , NISS score is better used to predict early mortality for polytrauma patients, while ISS score is not sufficient. Furthermore, when threshold probability rises between 0.5 and 0.7 , ISS score comes to be a better tool.

Previous studies have confirmed that initial arterial blood gases (ABGs, such as lactate and base excess) can be prognostic biomarkers in trauma, even in patients with normal vital signs ${ }^{[20-23]}$. ABGs are recognized as an important method to identify high-risk trauma patients. And variations in ABGs over time may guide early resuscitation, providing additional critical information. In this study, the odd ratios of lactate and base deficit were 1.27 and 0.95 with statistically significant. Nichol AD et al have proven that minor increases in lactate concentrations are associated with higher mortality rates in critically ill patients ${ }^{[24]}$. Janie Baxter et al. included 28 studies that recruited 44,154 adults in eight countries found that lactates were significantly higher in non-survivors than survivors ${ }^{[25]}$. When it comes to severely injured patients (ISS $\geq 15$ or $\geq 20$ with ICU stay), All these found lactate was significantly higher in non-survivors than survivors. More than a marker in acid-base disturbance, Paladino and colleagues has proven that with the addition of abnormal metabolic parameters with vital signs, the detection of major trauma increased significantly with sensitivity from $40.9-76.4 \%[26]$. Manuel Mutschler showed mortality rates in trauma patients rose from $7.4-51.5 \%$ with altered $B E$ values $^{[22]}$. Besides, $\mathrm{BE}$ indicates the acute presence of hypovolemic shock related to the need for hemostatic resuscitation, transfusion, and mortality, which were more appropriately than the current Advanced Trauma Life Support (ATLS) classification ${ }^{[22-23,26-28]}$. Based on the previous studies, we established a nomogram model to predict early mortality in polytrauma patients. Causal mediation analysis was applied to cover the limitations that if the variables were prompted by each other, and which variable might account for the beneficial effects of the model in our retrospective study. 4 independent risk factors: age, GCS score, lactate, and BE were included to this model eventually. 
Our nomogram model showed higher predictive performance in both train set and validation set than ISS or NISS scores. In addition, DCA analysis showed nomogram model over-perform better than NISS and ISS within 0.0 to 0.85 threshold probability. After that, to further improve the performance, a BP-ANN model was established. The BP-ANN model showed a higher F1 score, specificity, precision, AUC, and AUPRC than nomogram model. Besides, it works well in validation set too.

There are many strengths in the nomogram model together with web version. First, our nomogram model was based on four accessible parameters and an available in any level hospital in any country. Second, it is practical for physicians to evaluate polytrauma patients on admission with a nomogram model without any complicated calculation, even no calculation is needed on web version. Third, unlike ISS or NISS scoring or Nation Early Warning Scoring (NEWS), our nomogram model focused mainly on the homeostasis with ABGs and consciousness which reflects the status of central nervous system, while NEWS may not change significantly when homeostasis is disturbed. Fourth, nomogram model may predict the early death risk for individual patients, which is unlike the classic scoring system that focuses on a population of patients with the same physical or anatomical parameters, ignoring the status of individual patient. Although our nomogram model works well in polytrauma patients, a BP-ANN model was created to improve the GAS-TRS system further, inheriting the advantages of nomogram model and making it progress.

However, the retrospective nature of this study and single center study are clear limitations of this study, and the authors are aware of this shortcoming. We are in the process of conducting multiple centers studies to validate our study, and welcome scholars all over the world to examine our model in polytrauma patients.

\section{Conclusions}

In this large observational study, we established a practical GAS-TRS system to evaluate early mortality within 72 hours in polytrauma. GAS-TRS includes a quick nomogram model together with a web vision for quick evaluate high death risk and a precise BP-ANN model. GAS-TRS system is based on arterial blood gas which may reflect homeostasis and GCS score which reflects the status of central nervous system. This predictive system showed well performance and had a well net benefit in clinical decision-making. This scoring system might be important to identify high-risk trauma patients and even works much more than we know like guiding early resuscitation.

\section{Abbreviations}

DOA

dead on arrival

BP-ANN

back-propagation artificial neural network

PCT

procalcitonin

BMI

body mass index

ISS

injury severity score

GCS

Glasgow coma scale

RCS

Restricted cubic splines

HR 
hazard ratio

VIF

variance inflation factor

AUC

area under the curve

ROC

receiver operating characteristic

C-index

consistency index

DCA

decision curve analysis

CMA

Causal mediation analysis

ACME

average causal mediation effect

$\mathrm{ADE}$

average direct effect

ROCs

Receiver operator curves

AUPRC

area under the precision-recall curve

IQR

interquartile range

ABGs

arterial blood gases

ATLS

Advanced Trauma Life Support

NEWS

Nation Early Warning Scoring

BE

base excess

GAS-TRS

ABGs-Age-GCS based Triage and Risk Scoring system

\section{Declarations}

Ethics approval and consent to participate This retrospective population study was secondary analysis using unidentified data from the DRYAD database (https://datadryad.org), an open-access public database, obtained and from Dr. Halvachizadeh with formal authorization. Original studies were approved by regional institutional review board, and their public data included the anonymized patients which did not require further patient consent.

Consent for publication Not applicable.

Availability of data and materials Not applicable.

Competing interests The authors declare that they have no competing interests. 
Funding Not applicable.

Authors' contributions TX, SW and ND contributed equally to this work. TX, JT and XC conceptualized the research aims, planned the analyses, and guided the literature review. JW,XM,SL and LH processing the data and XZ is the statistician in our team and participated in performing the statistical analysis and reviewing the manuscript for statistical error. TX and ND wrote the first draft of the paper, and the other authors provided comments and approved the final manuscript.

Acknowledgements We thank Dr. Halvachizadeh and his team for their kindness of sharing polytrauma data on public website for re-use and supervision. We are very grateful for the formal authorization and permission from Dr. Halvachizadeh and continuous support.

\section{References}

1. Perel P, Prieto-Merino D, Shakur H, et al. Predicting early death in patients with traumatic bleeding: development and validation of prognostic model. BMJ. 2012;345: e5166.

2. Rhee P, Joseph B, Pandit V, et al. Increasing trauma deaths in the United States. Ann Surg. 2014;260(1):13-21.

3. Norton R, Kobusingye O. Injuries. N Engl J Med. 2013;368(18):1723-1730.

4. Ker K, Kiriya J, Perel P, Edwards P, Shakur H, Roberts I. Avoidable mortality from giving tranexamic acid to bleeding trauma patients: an estimation based on WHO mortality data, a systematic literature review and data from the CRASH-2 trial. BMC Emerg Med 2012;12(1):3.

5. Regel G, Lobenhoffer P, Grotz M, Pape HC, Lehmann U, Tscherne H. Treatment results of patients with multiple trauma: an analysis of 3406 cases treated between 1972 and 1991 at a German Level I Trauma Center. J Trauma Acute Care Surg. 1995; 38(1):70-8.

6. Tien HC, Spencer F, Tremblay LN, Rizoli SB, Brenneman FD. Preventable deaths from hemorrhage at a level I Canadian trauma center. J Trauma 2007; 62:142-6.

7. Domingues CA, Coimbra R, Poggetti RS, Nogueira LS, de Sousa RMC. New Trauma and Injury Severity Score (TRISS) adjustments for survival prediction. World J Emerg Surg. 2018; 13:12.

8. Oyeniyi BT, Fox EE, Scerbo M, Tomasek JS, Wade CE, Holcomb JB. Trends in 1029 trauma deaths at a level 1 trauma center: Impact of a bleeding control bundle of care. Injury. 2017;48(1):5-12.

9. AlRawahi AN, AlHinai FA, Doig CJ, et al. The prognostic value of serum procalcitonin measurements in critically injured patients: a systematic review. Crit Care. 2019;23(1):390. Published 2019 Dec 3.

10. Hamed R, Maaref A, Amira F, Aouni H, Mekki I, Jebali A. Prognostic value of scoring tools in severe trauma patients admitted to the emergency department. Tunis Med. 2018;96(3):203-208.

11. Wilson MS, Konda SR, Seymour RB, Karunakar MA; Carolinas Trauma Network Research Group. Early Predictors of Mortality in Geriatric Patients with Trauma. J Orthop Trauma. 2016;30(9): e299-e304.

12. Dezman ZD, Comer AC, Smith GS, Narayan M, Scalea TM, Hirshon JM. Failure to clear elevated lactate predicts 24hour mortality in trauma patients. J Trauma Acute Care Surg. 2015;79(4):580-585.

13. Halvachizadeh, Sascha (2019), Data from: How to detect a polytrauma patient at risk of complications: a validation and database analysis of four published scales, Dryad, Dataset, https://doi.org/10.5061/dryad.bnzs7h45v.

14. Halvachizadeh S, Baradaran L, Cinelli P, Pfeifer R, Sprengel K, Pape HC. How to detect a polytrauma patient at risk of complications: A validation and database analysis of four published scales. PLoS One. 2020 Jan 24;15(1):e0228082. doi: 10.1371/journal.pone.0228082.

15. Zhang Z, Zheng C, Kim C, Van Poucke S, Lin S, Lan P. Causal mediation analysis in the context of clinical research. Ann Transl Med. 2016;4(21):425. 
16. Driessen MLS, Sturms LM, van Zwet EW, Bloemers FW, Ten Duis HJ, Edwards MJR, den Hartog D, de Jongh MAC, Leenhouts PA, Poeze M, Schipper IB, Spanjersberg R, Wendt KW, de Wit RJ, van Zutphen SWAM, Leenen LPH. Evaluation of the Berlin polytrauma definition: A Dutch nationwide observational study. J Trauma Acute Care Surg. 2021 Apr 1;90(4):694-699.

17. Soni KD, Mahindrakar S, Gupta A, Kumar S, Sagar S, Jhakal A. Comparison of ISS, NISS, and RTS score as predictor of mortality in pediatric fall. Burns Trauma. 2017 Aug 8;5:25.

18. Smith BP, Goldberg AJ, Gaughan JP, Seamon MJ. A comparison of Injury Severity Score and New Injury Severity Score after penetrating trauma: A prospective analysis. J Trauma Acute Care Surg. 2015 Aug;79(2):269-74.

19. Eid HO, Abu-Zidan FM. New Injury Severity Score is a better predictor of mortality for blunt trauma patients than the Injury Severity Score. World J Surg. 2015 Jan;39(1):165-71.

20. Vincent JL, Quintairos E Silva A, Couto L Jr, Taccone FS. The value of blood lactate kinetics in critically ill patients: a systematic review. Crit Care. 2016 Aug 13;20(1):257.

21. Régnier MA, Raux M, Le Manach Y, Asencio Y, Gaillard J, Devilliers C, Langeron O, Riou B. Prognostic significance of blood lactate and lactate clearance in trauma patients. Anesthesiology. 2012 Dec;117(6):1276-88.

22. Mutschler M, Nienaber U, Brockamp T, Wafaisade A, Fabian T, Paffrath T, Bouillon B, Maegele M; TraumaRegister DGU. Renaissance of base deficit for the initial assessment of trauma patients: a base deficit-based classification for hypovolemic shock developed on data from 16,305 patients derived from the TraumaRegister DGUß. Crit Care. 2013 Mar 6;17(2):R42.

23. Raux M, Le Manach Y, Gauss T, Baumgarten R, Hamada S, Harrois A, Riou B, Duranteau J, Langeron O, Mantz J, Paugam-Burtz C, Vigue B; TRAUMABASE Group. Comparison of the Prognostic Significance of Initial Blood Lactate and Base Deficit in Trauma Patients. Anesthesiology. 2017 Mar;126(3):522-533.

24. Nichol AD, Egi M, Pettila V, Bellomo R, French C, Hart G, Davies A, Stachowski E, Reade MC, Bailey M, Cooper DJ. Relative hyperlactatemia and hospital mortality in critically ill patients: a retrospective multi-centre study. Crit Care. 2010;14(1):R25.

25. Baxter J, Cranfield KR, Clark G, Harris T, Bloom B, Gray AJ. Do lactate levels in the emergency department predict outcome in adult trauma patients? A systematic review. J Trauma Acute Care Surg. 2016 Sep;81(3):555-66.

26. Paladino L, Sinert R, Wallace D, Anderson T, Yadav K, Zehtabchi S. The utility of base deficit and arterial lactate in differentiating major from minor injury in trauma patients with normal vital signs. Resuscitation. 2008 Jun;77(3):363-8.

27. Nolan J. Fluid resuscitation for the trauma patient. Resuscitation. 2001 Jan;48(1):57-69.

\section{Figures}




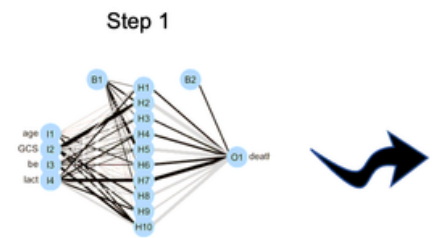

Initiate ANN

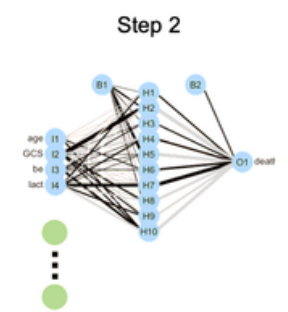

Inputs Layers Selection

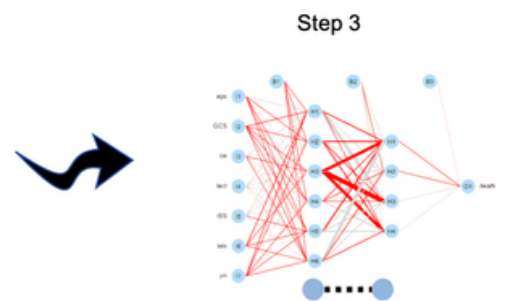

Hide Layers Selection

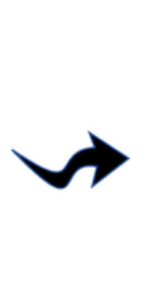

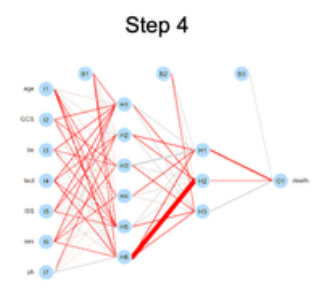

ANN Model
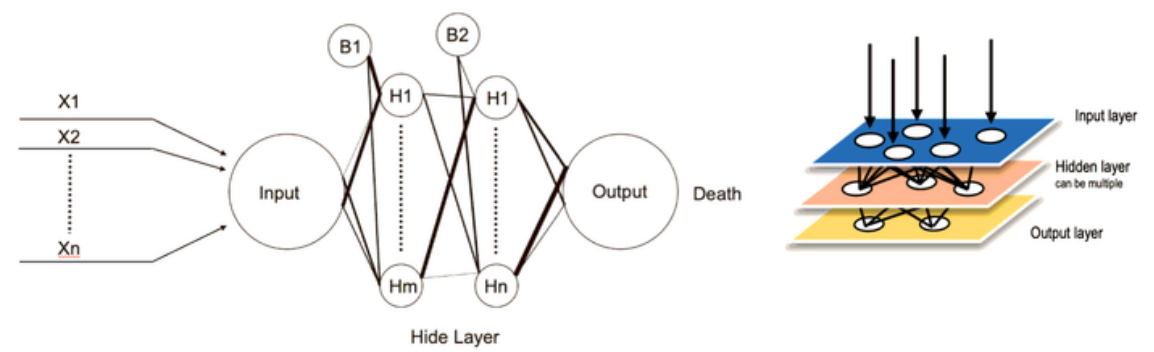

ANN Model Establishment

\section{Figure 1}

Scheme of BP-ANN establishmen.

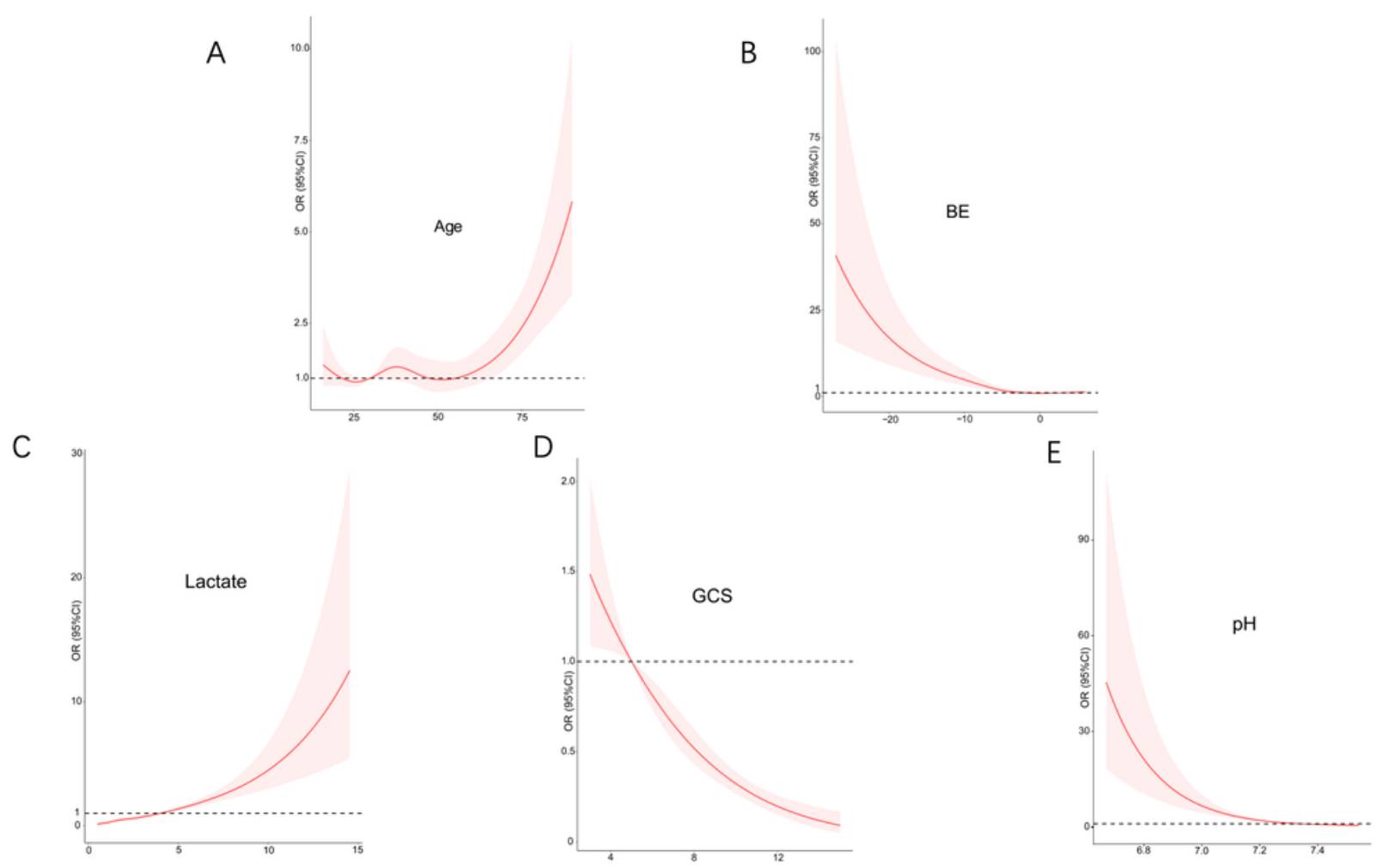

Figure 2 
Restricted Cubic Spline Regression of the Relationship Between independent factors and mortality. Lines are multiple adjusted odd ratios, with 95\% confidence intervals derived from restricted cubic spline regression. Reference lines for no association are indicated by the solid bold lines at a hazard ratio of 1.0. A-E. RCS based on pH, lactate acid, GCS, age and $\mathrm{BE}$.

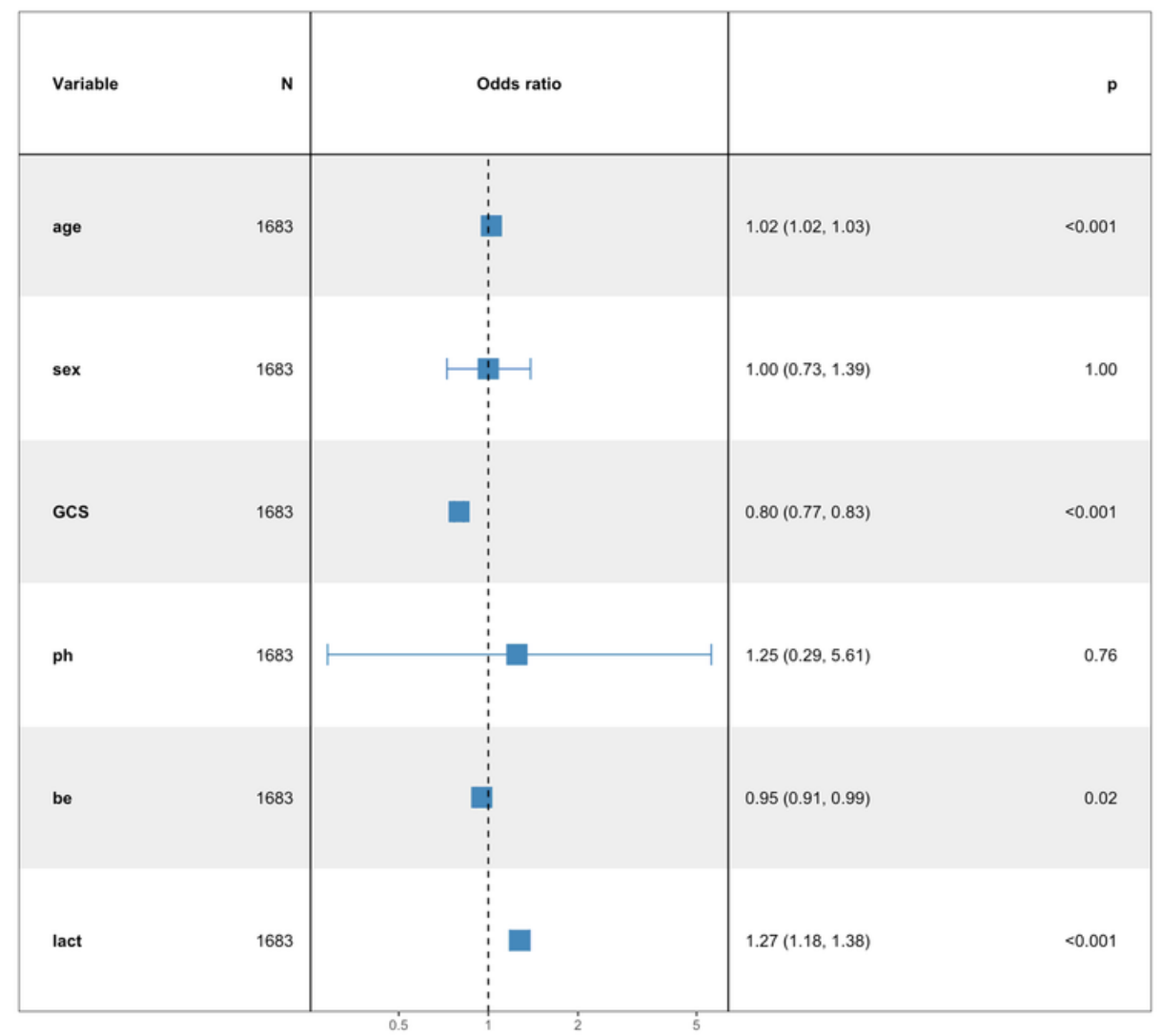

\section{Figure 3}

Multi-Variate Logistic Regression analysis for nomogram modeling. 
A

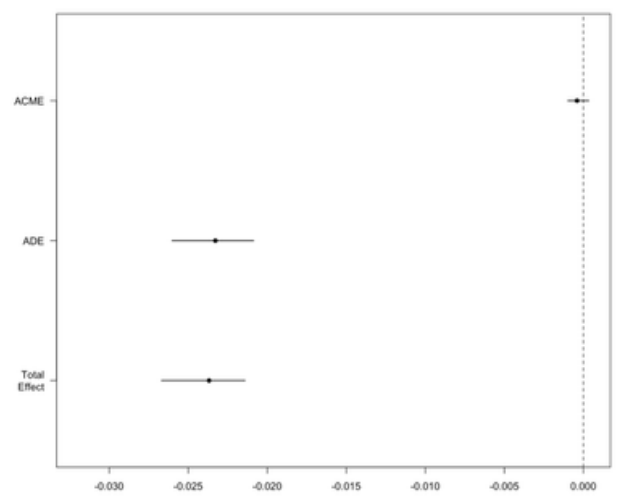

Lactate Acid
B

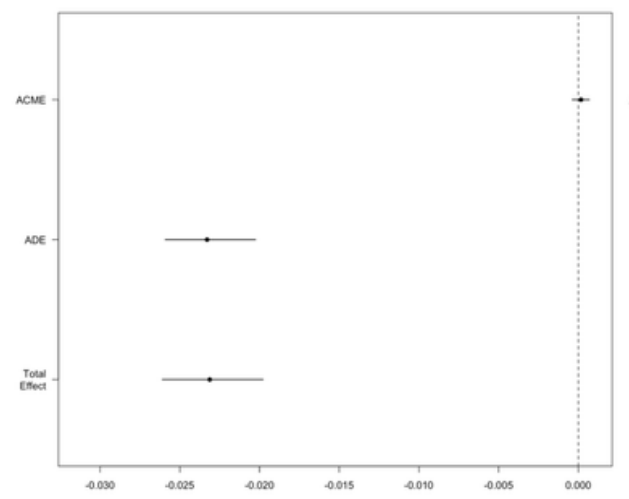

Age
C

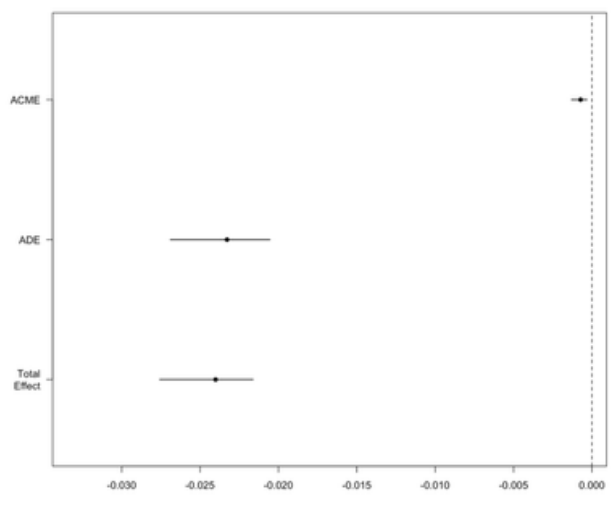

$\mathrm{BE}$

\section{Figure 4}

Causal mediation analysis for nomogram modeling. A-C: Lactated acid,age and BE. ACME: average causal mediation effect. ADE: average direct effect. 
Points

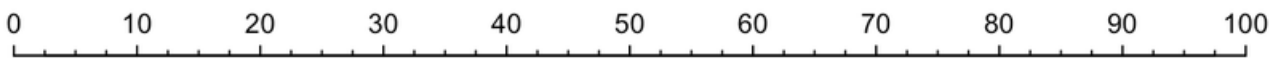

age

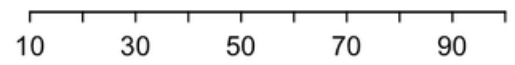

GCS

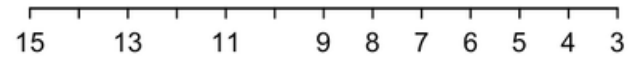

be

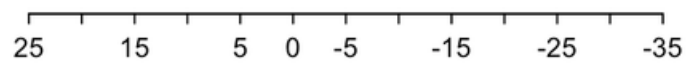

lact

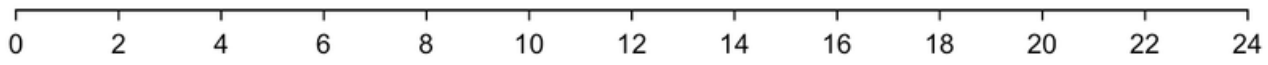

Total Points

\begin{tabular}{llllllllllll}
\hline 0 & 20 & 40 & 60 & 80 & 100 & 120 & 140 & 160 & 180 & 200 & 220
\end{tabular}

Polytrauma Mortality

$\begin{array}{llllllll}0.05 & 0.1 & 0.2 & 0.4 & 0.6 & 0.8 & 0.9 & 0.95\end{array}$

\section{Figure 5}

Nomogram for early mortality prediction in polytraumatic patients. The higher total points indicate the lower expected survival. 
A

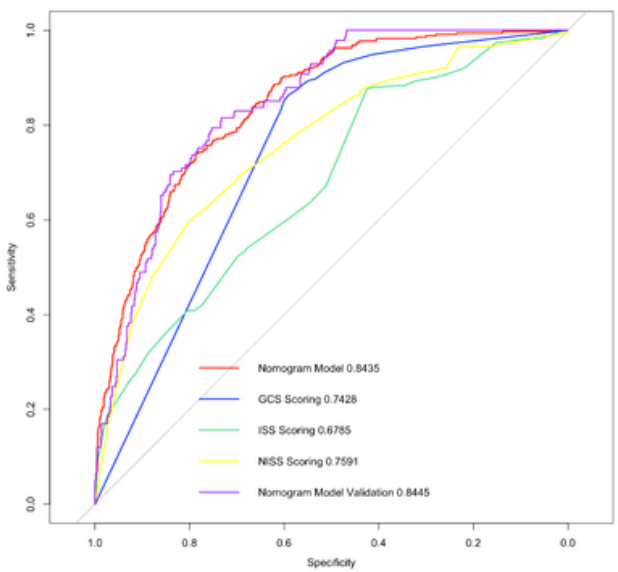

B

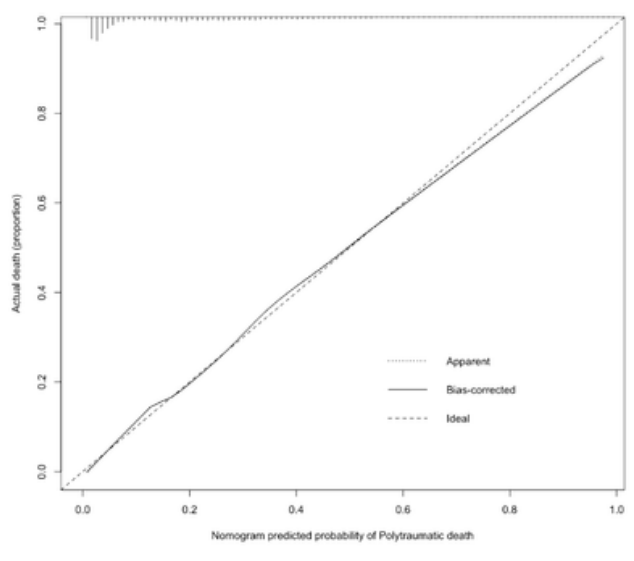

C

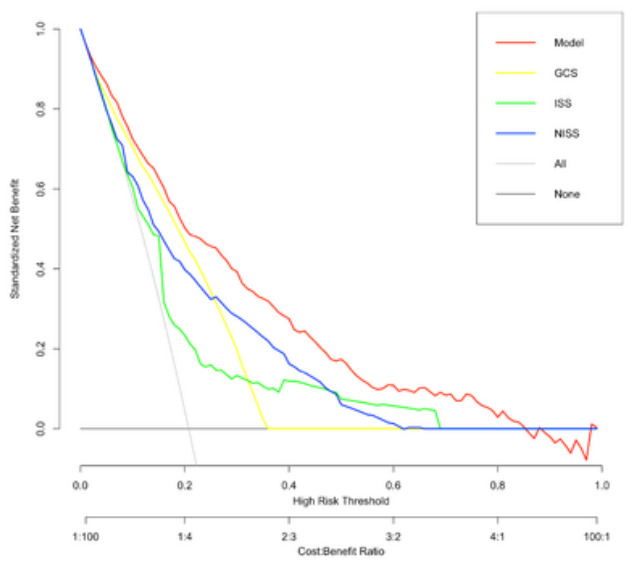

Figure 6

Evaluation of the pefomance of nomogram model. A. Receiving operating characteristics curves of Nomogram predictive model and ISS and validation set.B. Calibration curve of Nomogram in training. C. Decision curve analysis of Nomogram predictive model and ISS.

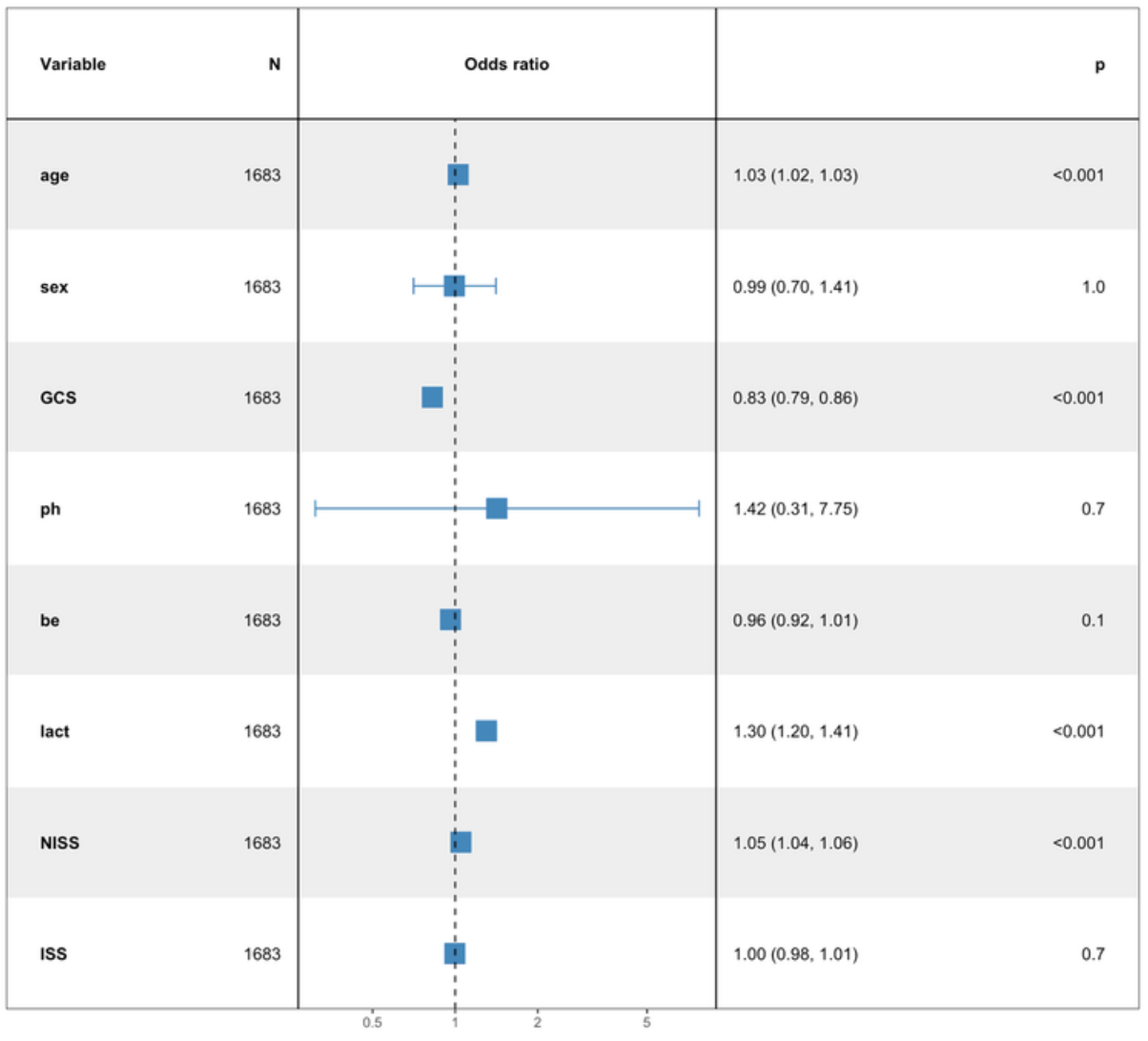

Page $16 / 19$ 


\section{Figure 7}

Multi-Variate Logistic Regression analysis for BP-ANN modeling.

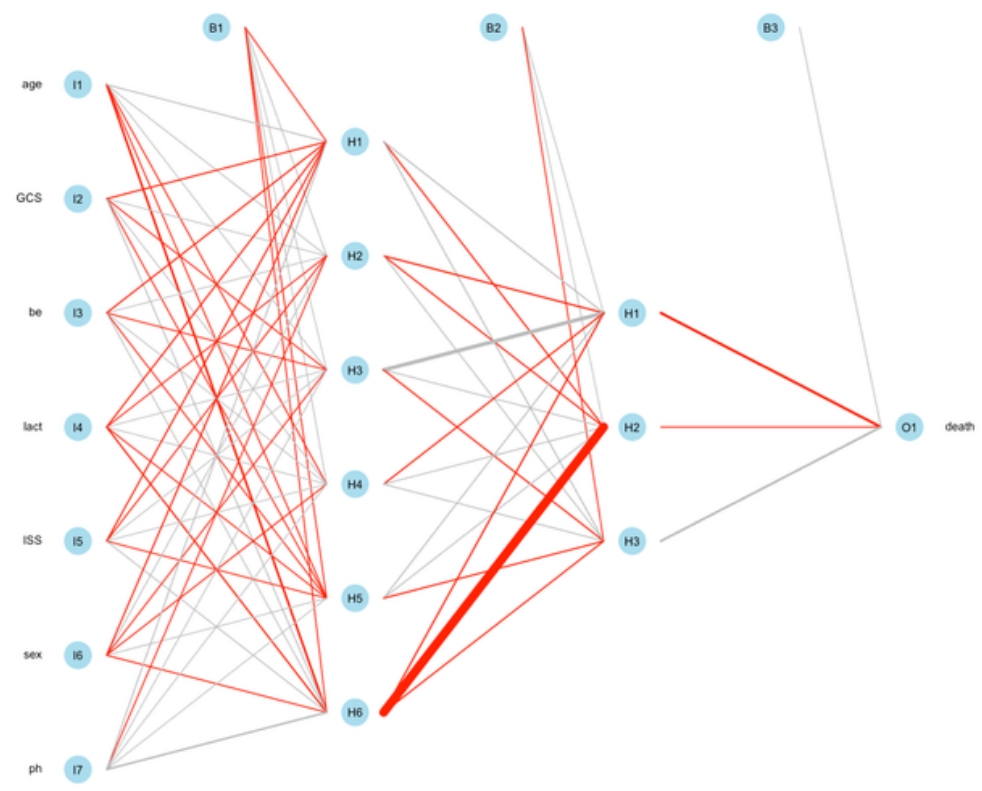

\section{Figure 8}

BP-ANN for early mortality prediction in polytraumatic patients. Ix: input neuron. Hx: hiden neuron. Ox:output neuron. Bx: threshold. 


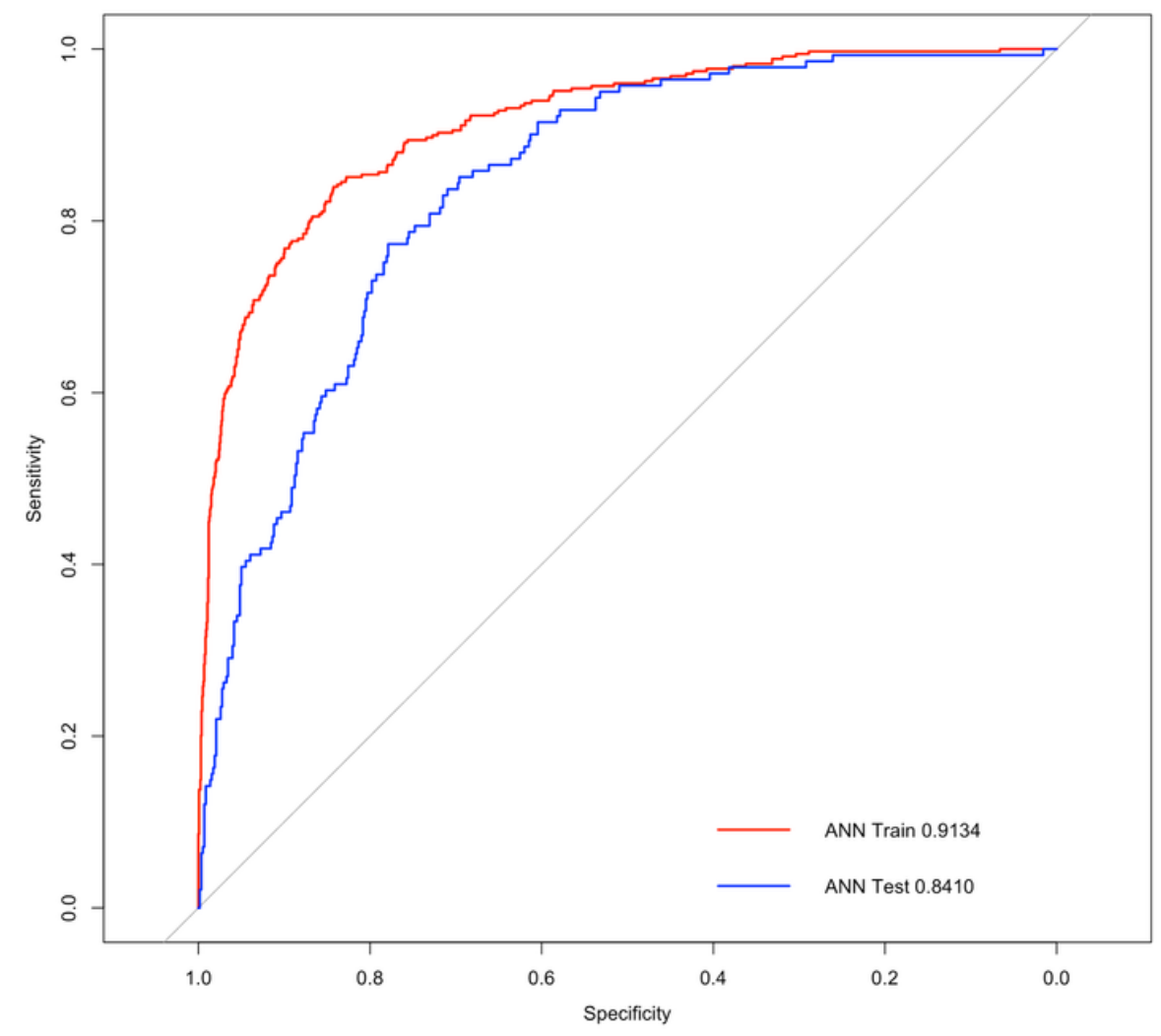

Figure 9

Receiving operating characteristics curves of BP-ANN predictive model in train and validation set.

A

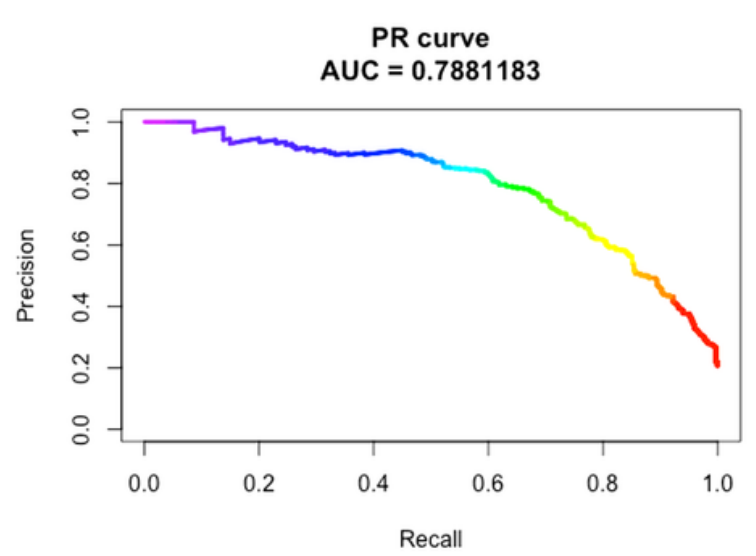

B

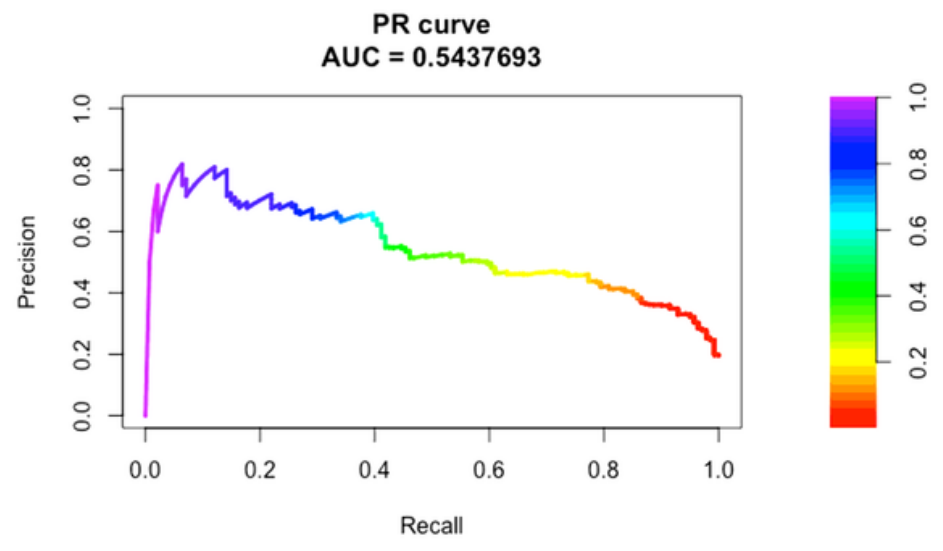

Figure 10 
Precision-recall curves of BP-ANN predictive in train and validation set.

Page 19/19 